\title{
Hiperaldosteronismo Primário Causado por Aldosteronoma: Problemas no Diagnóstico Etiológico
}

\section{caso especial}

\section{Debora lucia S. Danilovic Daniel SoARes Freire Diane Belchior Paraíba Rodrigo A. BRANDÃo Neto ANTÔNIO MARMO LUCON maria adelaide A. Pereira}

Serviço de Endocrinologia e Metabologia do Hospital das Clínicas da Faculdade de Medicina da USP (HCFMUSP), São Paulo, SP.

\section{RESUMO}

O hiperaldosteronismo primário (HAP) representa importante causa de hipertensão arterial secundária, potencialmente curável, que tem recebido atenção recente em função do aumento de sua prevalência desde a introdução do rastreamento pelo uso da relação aldosterona/atividade plasmática de renina. Apresentamos caso de HAP causado por provável aldosteronoma, coexistente com adenoma adrenal não-funcionante contra-lateral, o que dificultou o diagnóstico etiológico. Discutimos as formas mais apropriadas de rastrear, confirmar o diagnóstico de HAP e diferenciar as suas diversas etiologias, com destaque para o papel do cateterismo de adrenais no diagnóstico diferencial definitivo entre aldosteronoma e hiperaldosteronismo idiopático, com implicações no sucesso terapêutico. (Arq Bras Endocrinol Metab 2007;51/3:478-487)

Descritores: Hiperaldosteronismo primário; Aldosteronoma; Hiperaldosteronismo idiopático; Hipocalemia; Hipertensão arterial

\section{ABSTRACT}

Primary Aldosteronism Caused by Aldosteronoma: Problems in the Etiologic Diagnosis.

Primary aldosteronism (PA) represents an important cause of secondary hypertension, potentially curable, and it has been receiving particular attention due to its increasing prevalence, after the beginning of the use of plasma aldosterone concentration to plasma renin activity ratio as a screening method. We present a case of PA caused by an aldosteronoma associated with a contralateral nonfunctioning adrenal adenoma, which resulted in difficulties in the final diagnosis. We discuss the most appropriated tests to screen, confirm the diagnosis of PA and define the etiology of the disorder, especially the adrenal veins sampling to distinguish the aldosteronoma and idiopathic hyperaldosteronism and to guide successful treatment. (Arq Bras Endocrinol Metab 2007;51/3:478-487)

Keywords: Primary aldosteronism; Aldosteronoma; Idiopathic hyperaldosteronism; Hypokalemia; Arterial hypertension

\section{Debora Lucia Seguro Danilovic (Residente de 20 ano do Serviço de Endocrinologia e Metabologia do HCFMUSP)}

JESTa APRESENTAÇão, vamos discutir problemas que podemos enfrentar no diagnóstico etiológico do hiperaldosteronismo primário (HAP). Para tanto, apresentamos o caso de uma paciente com aldosteronoma, na qual o diagnóstico topográfico do tumor adrenal foi problemático devido à coexistência de um adenoma não-funcionante em uma adrenal e de um provável adenoma contra-lateral, produtor de aldosterona.
Recebido em 28/04/05

Revisado em 29/11/05

Aceito em 26/12/06 


\section{APRESENTAÇÃO DO CASO}

Paciente do sexo feminino, 50 anos, com diagnóstico de hipertensão arterial há 3 anos. Há 3 semanas apresentava desenvolvimento de cansaço e fraqueza em membros inferiores, de caráter progressivo, que culminou em paralisia dos quatro membros. A avaliação clínica e laboratorial inicial revelou hipertensão arterial $(\mathrm{PA}=180 \times 100$ $\mathrm{mmHg}$ ), tetraparesia, ECG com alteração de repolarização ventricular e presença de onda $U$, hipocalemia $(\mathrm{K}=1,4 \mathrm{mEq} / \mathrm{L})$, elevação de creatinofosfoquinase $(\mathrm{CPK}=19556 \mathrm{U} / \mathrm{L})$, aldosterona $(\mathrm{A})=23 \mathrm{ng} / \mathrm{dL}$, atividade de renina plasmática $(\mathrm{ARP})=<0,2 \mathrm{ng} / \mathrm{mL} /$ hora, relação $\mathrm{A} / \mathrm{ARP}=115 \mathrm{ng} / \mathrm{dL} / \mathrm{ng} / \mathrm{mL} / \mathrm{h}$. Os níveis de potássio foram corrigidos e a paciente foi novamente investigada para o diagnóstico de HAP, sem o uso de medicações hipotensoras. A nova determinação da relação A/ARP revelou valor de 40,8 ng/dL/ng/mL $/ \mathrm{h}$ e a aldosterona sérica, após a administração endovenosa de $2 \mathrm{~L}$ de soro fisiológico em 4 horas, foi de 7,7 ng/dL. Não houve redução da aldosterona durante o dia, embora tenha ocorrido queda esperada nos níveis de cortisol. A avaliação radiológica, realizada pela tomografia computadorizada (TC), revelou tumor de $1,8 \mathrm{x}$ $1,2 \times 1,2 \mathrm{~cm}$ na adrenal direita e adrenal esquerda normal ou discretamente aumentada (figura 1). Foi submetida ao cateterismo de veias adrenais, que revelou gradiente de aldosterona na adrenal esquerda (figura 1). Portanto, tratava-se de uma paciente com HAP, com tumor na adrenal direita, mas com cateterismo indicando produção unilateral de aldosterona à esquerda. Devido ao quadro de calculose biliar sintomática, houve indicação, com relativa urgência, de colecistectomia. Foi optado pela retirada do tumor da adrenal direita no mesmo tempo cirúrgico, na expectativa de que isso pudesse corrigir o quadro de hipertensão caso houvesse ocorrido algum erro no cateterismo. Entretanto, após a cirurgia, a paciente permaneceu hipertensa, com aldosterona elevada e renina bloqueada, confirmando a hipótese, levantada pelo cateterismo, de tumor na adrenal esquerda. $\mathrm{O}$ diagnóstico anátomo-patológico do tumor adrenal direito foi de adenoma (figura 2). A paciente foi medicada com espironolactona e amlodipina e permanece, há 2 anos, assintomática com controle adequado dos níveis de potássio e da pressão arterial.

\section{DISCUSSĀO}

O HAP pode ser definido como um estado de produção excessiva e relativamente autônoma de aldosterona. Nessas condições, inicia-se, nos túbulos renais, processo de retenção de sódio e água que, com o tempo, leva à supressão da produção da renina, hipertensão arterial e hipocalemia estabelecida ou tendência a hipocalemia (1-3).

A hipertensão arterial (HAS) é a principal manifestação do HAP, sendo que poucos pacientes podem

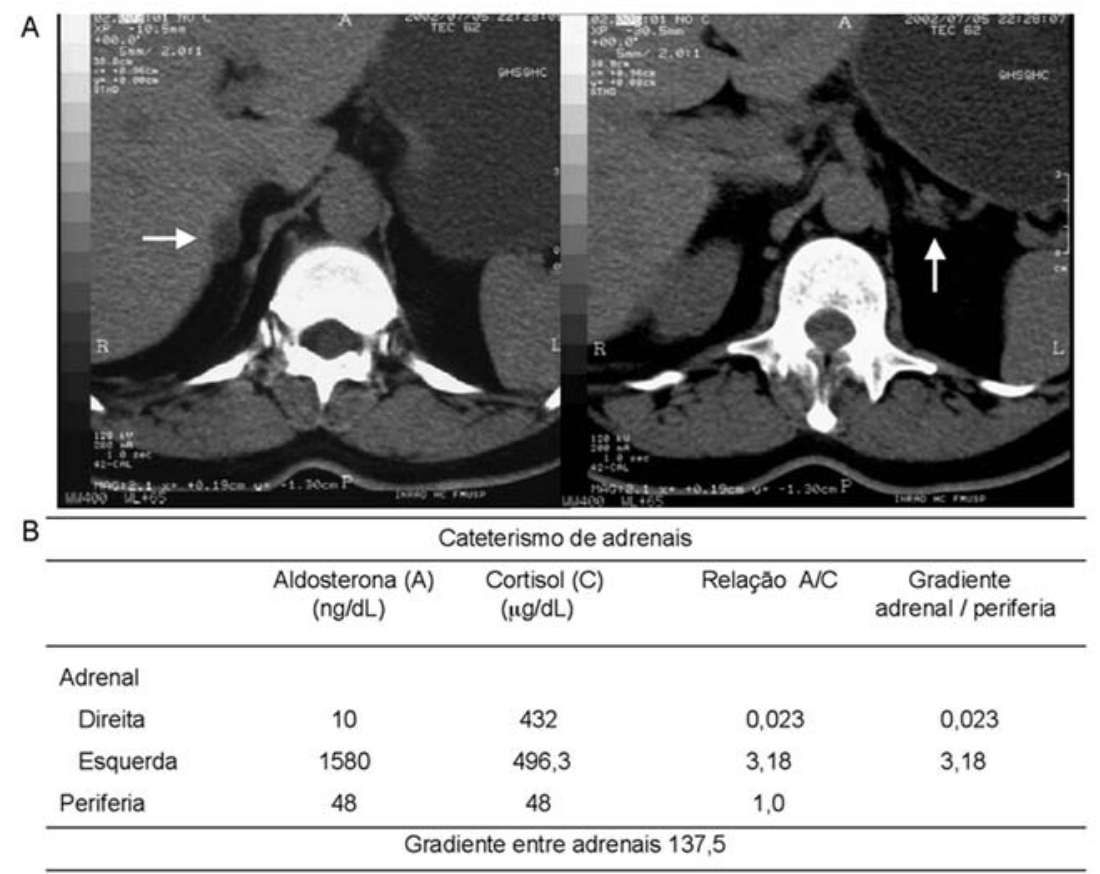

Figura 1. (A) Tomografia computadorizada de adrenais: nódulo à direita e espessamento discutível da adrenal esquerda. (B) Cateterismo de veias adrenais. 


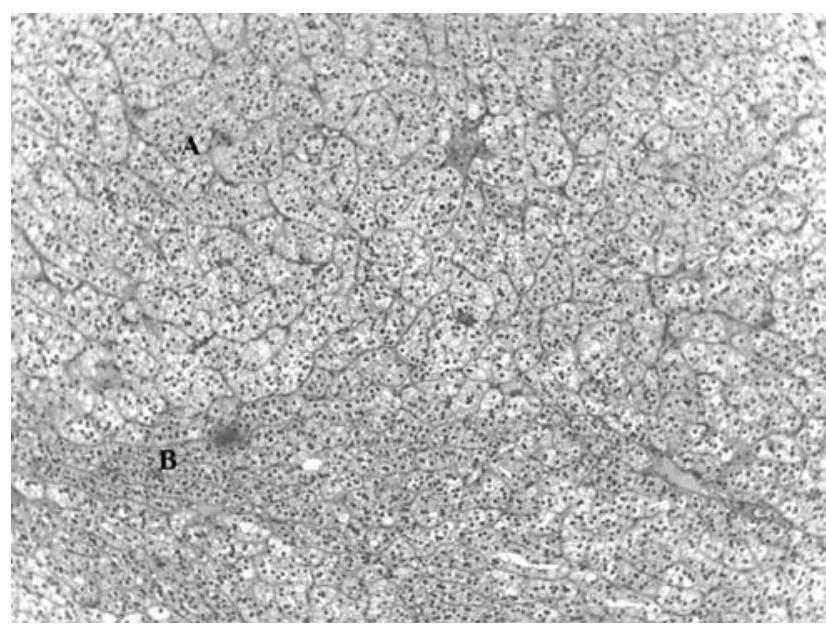

Figura 2. Histopatologia do tumor da adrenal ressecado da paciente: (A) adenoma adrenal. (B) Tecido adrenal normal.

ser normotensos. Alguns dados bioquímicos podem sugerir o diagnóstico de HAP nos pacientes hipertensos, como hipocalemia $(<3,5 \mathrm{mEq} / \mathrm{L})$ associada a excreção urinária aumentada de potássio (> $30 \mathrm{mEq} /$ dia $)$. Entretanto, uma porcentagem importante dos pacientes não tem diminuição nos níveis séricos de potássio e, portanto, a presença de hipocalemia não é considerada parâmetro com sensibilidade para o rastreamento de HAP. Embora a sua existência seja sugestiva, não devemos nos basear apenas na sua presença para investigar o hiperaldosteronismo em pacientes hipertensos. Outros dados bioquímicos sugestivos são alcalose metabólica, que acompanha a hipocalemia, natremia no limite superior da normalidade, hipomagnesemia e intolerância a glicose, esta última provocada pela diminuição da secreção de insulina em função dos níveis séricos de potássio diminuídos.

A nossa experiência com tumor adrenal unilateral produtor de aldosterona foi sistematizada pela análise retrospectiva de 29 pacientes com este diagnóstico, comprovado através de cirurgia, que foram acompanhados nos últimos 20 anos nos Serviços de Endocrinologia e Metabologia, de Nefrologia e de Urologia do HCFMUSP. Com relação ao sexo, pudemos observar uma preponderância absoluta do sexo feminino em relação ao masculino de $76 \%$ versus $24 \%$. A idade média dos pacientes foi de $43 \pm 11$ anos. A duração da hipertensão arterial até o diagnóstico variou de 1 a 35 anos, com uma mediana de 8 anos. Isto sugere que a investigação de HAP deve ser feita independentemente do tempo de duração da história clínica. Com relação aos sintomas apresentados por nossos pacientes, observamos sintomas decorrentes da hipertensão e da hipocalemia: 73\% apresentavam cefaléia, $43 \%$, paresia ou fraqueza muscular $(60 \%$ deles com tetraparesia e $40 \%$ com paraparesia), $42 \%$, cãimbras, $36 \%$, parestesias e poucos pacientes tiveram paralisia muscular, como a apresentada pela paciente em questão. Chamamos a atenção para o fato de que todos os nossos pacientes, exceto um que vinha em uso de espironolactona, tinham níveis séricos de potássio baixos. A média dos valores de potássio foi de 2,3 $\pm 0,5 \mathrm{mEq} / \mathrm{L}(1,5$ a $3,8 \mathrm{mEq} / \mathrm{L}$; mediana de 2,3 $\mathrm{mEq} / \mathrm{L})$. A gravidade da HAS foi variável, com valores de pressão sistólica de 150 a $240 \mathrm{mmHg}$ e de diastólica de 80 a $150 \mathrm{mmHg}$, com mediana de 180 x 110 $\mathrm{mmHg}$. Portanto, tivemos pacientes com hipertensão leve, moderada e grave. A maioria dos nossos pacientes necessitava de mais de dois agentes anti-hipertensivos para controle da PA, e vários pacientes permaneciam com PA média elevada mesmo usando número superior a duas drogas. Com relação à repercussão da hipertensão arterial, observamos que $88 \%$ dos nossos pacientes apresentavam retinopatia hipertensiva, $57 \%$, hipertrofia de ventrículo esquerdo (VE) no ecocardiograma e 50\%, sobrecarga de VE no ECG. Essa alta freqüência de alterações em órgãos-alvo talvez exemplifique a ação danosa da aldosterona sobre os vasos e coração, já bastante relatada na literatura (4-8).

O caso apresentado é bastante sugestivo do diagnóstico de HAP, já que o quadro clínico era de HAS, hipocalemia grave e paralisia muscular. Existia, portanto, necessidade óbvia de se proceder à investigação, que deve ser feita, inicialmente, por meio de um teste de rastreamento. O mais utilizado atualmente é o estudo da relação aldosterona/renina, mais especificamente aldosterona/atividade de renina plasmática (A/ARP). A simples combinação de níveis elevados, ou mesmo normais de aldosterona, frente a níveis reduzidos da atividade de renina plasmática é evidência de secreção autônoma de aldosterona e indica a possibilidade do diagnóstico de HAP. Uma relação A/ARP maior que 20 ou $35 \mathrm{ng} / \mathrm{dL} / \mathrm{ng} / \mathrm{mL} / \mathrm{h}$, com aldosterona plasmática maior que 15 ou $20 \mathrm{ng} / \mathrm{dL}$, é bastante sugestiva de HAP, com sensibilidade variando entre 90 e $100 \%(1,2,9-11)$. No nosso serviço consideramos um relação $\mathrm{A} / \mathrm{ARP} \geq 30 \mathrm{ng} / \mathrm{dL} / \mathrm{ng} / \mathrm{mL} / \mathrm{h}$, com aldosterona $>15 \mathrm{ng} / \mathrm{dL}$, indicativa do diagnóstico de HAP.

A utilização dessa relação é método sensível no diagnóstico de HAP, mas não tem boa especificidade e, por este motivo, a maioria dos autores conclui que ela deve ser utilizada apenas no rastreamento e nunca no diagnóstico definitivo de $\operatorname{HAP}(6,9,12,13)$. Entretanto, alguns investigadores acreditam que valores muito elevados dessa relação seriam suficientes para o diagnóstico definitivo (14). Um ponto que deve ser 
levado em consideração na interpretação da relação A/ARP é que vários medicamentos podem interferir na sua acurácia $(15,16)$. Assim, beta-bloqueadores elevam a relação e a maioria dos outros agentes hipotensores (diuréticos, inibidores da ECA e de receptores de angiotensina, bloqueadores de canais de cálcio, bloqueadores de receptores alfa-adrenérgicos) a diminuem e, portanto, podem interferir na interpretação dos resultados. Lembrar, ainda, que a espironolactona aumenta a renina e pode ou não aumentar a aldosterona interferindo, de maneira importante, na relação A/ARP. O ideal é fazer o estudo sem o uso dessas medicações ou utilizando medicamentos que interfiram menos com o teste, como os alfa-bloqueadores. Isso não quer dizer que a relação não possa ser interpretada na vigência dessas drogas. Por exemplo, uma relação elevada na vigência de medicações que tendem a diminuí-la é um dado bastante sugestivo de HAP, mas essa mesma relação aumentada com o uso de betabloqueadores pode representar um falso-positivo. $\mathrm{O}$ uso de antagonistas de receptor de mineralocorticóide afeta definitivamente o diagnóstico e, portanto, esses agentes hipotensores não devem ser utilizados durante os testes diagnósticos $(15,16)$.

A paciente apresentava uma relação A/ARP de $40,8 \mathrm{ng} / \mathrm{dl} / \mathrm{ng} / \mathrm{ml} / \mathrm{h}$, bastante sugestiva do diagnóstico de HAP. Os dados relativos a aldosterona, atividade de renina plasmática e relação A/ARP dos nossos 29 pacientes com aldosteronoma estão demonstrados na figura 3 . Observamos que a mediana da aldosterona foi alta, em torno de $56 \mathrm{ng} / \mathrm{dL}$, com a maioria dos pacientes apresentando valores maiores que $25 \mathrm{ng} / \mathrm{dL}$, embora um deles tivesse aldosterona de $12 \mathrm{ng} / \mathrm{dL}$. A atividade de renina plasmática foi baixa na grande maioria de nossos pacientes, com valores indetectáveis ou inferiores a $0,8 \mathrm{ng} / \mathrm{mL} /$ hora, exceto em dois pacientes que estavam tomando espironolactona por ocasião do exame. Com exceção desses dois pacientes e da paciente apresentada, observamos que todos tiveram relação $\mathrm{A} / \mathrm{ARP}$ superior a 50 , sendo a maioria acima de $100 \mathrm{ng} / \mathrm{dl} / \mathrm{ng} / \mathrm{ml} / \mathrm{h}$. Observem que a espironolactona aumenta a renina e, como a produção de aldosterona na presença do tumor é independente da renina, a relação $\mathrm{A} / \mathrm{ARP}$ diminui bastante.

Portanto, na nossa paciente, o rastreamento foi claramente positivo e ela foi submetida a um teste confirmatório do diagnóstico de HAP. A manobra que nós utilizamos foi a infusão endovenosa de solução salina. No indivíduo normal, a sobrecarga de sal diminui, através do bloqueio do SRA, a produção de aldosterona; no hiperaldosteronismo isso não ocorre porque o SRA já está total ou parcialmente bloqueado. A aldosterona foi determinada antes e após a infusão de $2 \mathrm{~L}$ de soro fisiológico $0,9 \%$ (500 mL/hora). O teste foi realizado no período da manhã e coletou-se material para dosagem de aldosterona às 8 e às 12 horas, após a infusão dos dois litros de soro. Um valor de aldosterona menor que $5 \mathrm{ng} / \mathrm{dL}$ exclui o diagnóstico de hiperaldosteronismo primário (13). O nível sérico de potássio da nossa paciente foi corrigido antes do teste e monitorado durante a infusão salina. Este é um cuidado que devemos ter porque o aumento da oferta de sódio ao túbulo renal distal pode levar à excreção aumentada de potássio e hipocalemia severa.

O diagnóstico de HAP foi confirmado na nossa paciente, que apresentou aldosterona de $7,7 \mathrm{ng} / \mathrm{dl}$
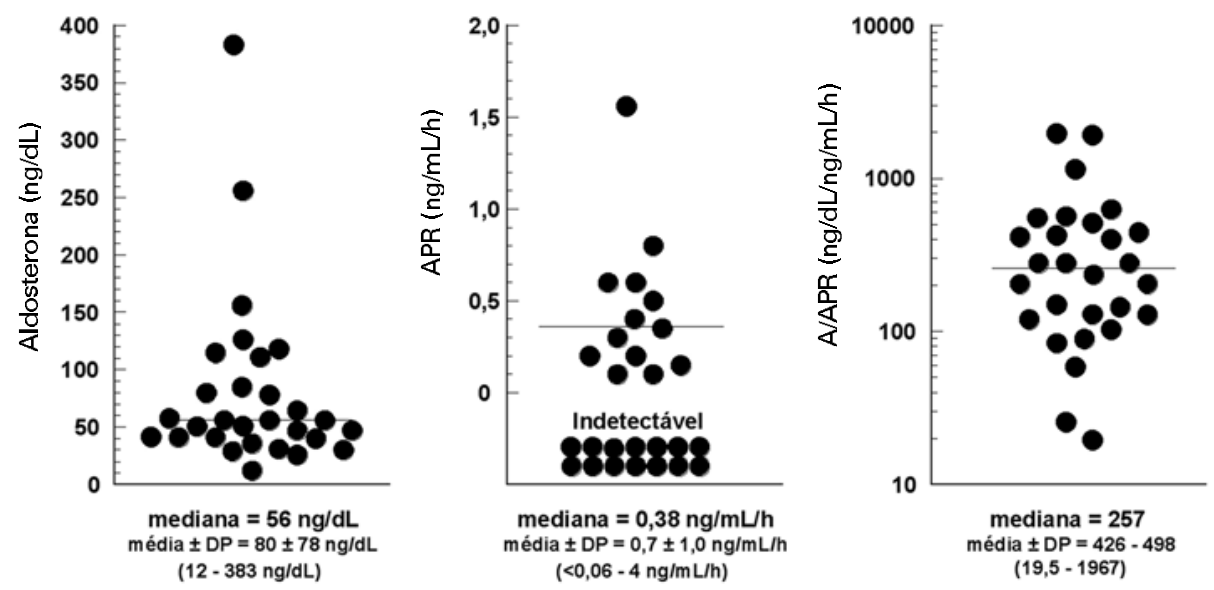

Figura 3. Aldosterona plasmática, atividade plasmática de renina e relação aldosterona/atividade plasmática de renina nos casos de adenomas unilaterais produtores de aldosterona do Serviço de Endocrinologia e Metabologia do HCFMUSP. 
após a infusão salina. Vinte dos nossos pacientes realizaram o teste de infusão salina e em nenhum deles houve queda da aldosterona para valores inferiores a 5 $\mathrm{ng} / \mathrm{dL}$. De fato, a maioria não apresentou alteração nos níveis do hormônio após a infusão salina. A queda apresentada por alguns pacientes pode refletir variações da secreção tumoral ou o ritmo circadiano da aldosterona, que é o mesmo do ACTH em pacientes com aldosteronoma.

Uma vez confirmada a presença de HAP na nossa paciente, passamos para a última etapa do diagnóstico que é o esclarecimento da etiologia do HAP. As principais causas são o aldosteronoma ou adenoma produtor de aldosterona (APA), em que a produção de aldosterona é unilateral, e o hiperaldosteronismo idiopático (HAI), também chamado de hiperplasia bilateral das adrenais, porque a produção de aldosterona é bilateral.

O aldosteronoma é um tumor do córtex adrenal, em geral autônomo, isto é, a produção de aldosterona não é influenciada pelo sistema renina angiotensina. Nessa doença, as manobras funcionais, que tendem a alterar a renina, em geral não influenciam a produção de aldosterona. Entretanto, cerca de $20 \%$ dos aldosteronomas podem responder a angiotensina e, portanto, respondem a manobras que elevam a renina, como a posição ortostática. A maioria dos aldosteronomas responde ao ACTH e, dessa forma, a produção de aldosterona segue o ritmo circadiano de secreção de cortisol, diminuindo no decorrer do dia $(1,2,10,11)$. Nesses casos de hiperaldosteronismo, a cirurgia cura a hipertensão em cerca de 40 a $50 \%$ dos casos e corrige a hipocalemia na sua totalidade.

O hiperaldosteronismo idiopático é uma patologia em que existe hiperplasia bilateral das adrenais e representa atualmente 50 a $60 \%$ dos HAP. A produção de aldosterona responde ao estímulo do SRA e, portanto, manobras que estimulam a renina podem estimular a produção de aldosterona. Entretanto, alguns pacientes podem não responder a essas manobras. A produção de aldosterona não segue o ritmo de secreção de cortisol. A cirurgia cura apenas uma minoria desses pacientes e, portanto, não está indicada nesses casos, que devem ser tratados clinicamente. Os pacientes portadores de HAI apresentam, em geral, níveis mais baixos de aldosterona e têm, com menor frequiência, hipocalemia $(1,2,10,11)$.

Uma terceira causa rara de HAP é a hiperplasia primária da adrenal (HPA), que é uma patologia que se comporta funcionalmente como o aldosteronoma e que também responde bem à cirurgia. O hiperaldosteronismo primário também pode estar associado ao car- cinoma de adrenal, mas essa é uma eventualidade rara, que deve ser aventada nos casos de HAP com tumores adrenais grandes, maiores que $4 \mathrm{~cm}$, e nesses casos ocorre, em geral, a produção de outros hormônios do córtex adrenal. Finalmente, nós temos o HAP familiar, tipo I e II. No HAP tipo I, a produção de aldosterona é diminuída pela administração de glicocorticóides (GRA). Trata-se de doença autossômica dominante, que deve ser pesquisada quando existe história familiar de hipertensão e hipocalemia que se instala precocemente no decorrer da vida. Nessa doença existe a expressão de um gene quimérico, que faz com que a zona fasciculada da adrenal possa sintetizar aldosterona e, como essa região do córtex adrenal está sob o controle do ACTH, vamos ter um hiperaldosteronismo responsível ao glicocorticóide, já que o ACTH é o grande regulador dessa camada da glândula. Essa é uma doença rara que pode ser tratada com baixas doses de glicocorticóides. O HAP familiar tipo II é muito raro e se refere à ocorrência de APA e HAI numa mesma família $(2,10,11,17,18)$.

O que devemos ter em mente é que $95 \%$ dos pacientes com HAP são portadores de APA ou HAI. É essencial o estabelecimento desse diagnóstico diferencial porque no aldosteronoma a cirurgia corrige a hipocalemia e pode resolver a hipertensão arterial, enquanto ela é ineficaz na hiperplasia bilateral.

Qual a freqüência relativa dessas duas doenças? Vários estudos têm demonstrado que, atualmente, o hiperaldosteronismo idiopático representa, ao contrário do que se acreditava anteriormente, a principal causa de HAP (19-21).

Algumas características demográficas, clínicas e laboratoriais são mais freqüentes em um ou outro grupo. Desta forma, a idade média dos pacientes com aldosteronoma é em torno de 45 anos, enquanto a dos pacientes com HAI, em geral, é superior a 50 anos. No primeiro grupo predomina o sexo feminino e no segundo, o masculino. A hipertensão e a hipocalemia tendem a ser mais graves no aldosteronoma e os níveis da aldosterona são mais elevados neste grupo. Entretanto, existe uma sobreposição grande entre os dois grupos, de modo que essas características não permitem o diagnóstico diferencial definitivo entre as duas doenças (2).

O diagnóstico diferencial definitivo é baseado em dados laboratoriais, radiológicos e, principalmente, como veremos a seguir, no cateterismo das veias adrenais. Como pacientes portadores de APA tendem a não responder a renina, ao contrário dos portadores de HAI, podemos diferenciá-los através de manobras que elevem a renina. Uma dessa manobras é o teste postural, que é feito determinando-se a atividade plas- 
mática de renina e a aldosterona antes e após a adoção de postura ortostática por, no mínimo, duas horas. Um indivíduo normal ou com HAI responde com aumento da renina e da aldosterona. No APA, a renina e a aldosterona não se elevam, ou ainda, em alguns pacientes, a aldosterona cai seguindo o ritmo de cortisol. Considera-se resposta positiva ao teste postural elevação maior que 30\% dos níveis de aldosterona. No HAI temos, em geral, elevação acima de 35\% (1). Devemos fazer a determinação concomitante dos níveis de cortisol, que normalmente devem cair no decorrer do dia. Se houver elevação do cortisol devemos subtrair seu incremento percentual daquele da aldosterona. Entretanto, como já dissemos anteriormente, alguns pacientes com HAI não respondem à renina e alguns pacientes com APA podem responder; assim, o diagnóstico diferencial não deve se basear apenas em testes funcionais, embora eles forneçam dados adicionais para a diferenciação definitiva.

Outra forma de diagnóstico diferencial funcional é a resposta a espironolactona. Após a administração deste antagonista de receptor de mineralocorticóide, a renina se eleva. Na maioria dos casos de APA, não se observa aumento da aldosterona plasmática; se houver elevação, é mais provável tratar-se de HAI.

Dos nossos 29 pacientes com aldosteronoma, 21 foram submetidos ao teste postural. Destes, 5 pacientes (23\%) responderam com elevação da aldosterona maior que $35 \%$, um paciente teve aumento intermediário de $32 \%$ e o restante $(72 \%)$ não respondeu. Embora a falta de resposta prevaleça, nós verificamos que o teste não tem acurácia para a diferenciação.

Atualmente, os testes mais recomendados para a realização do diagnóstico diferencial são os exames de imagem, tomografia computadorizada (TC) ou ressonância magnética (RM) das adrenais, e o cateterismo com coleta seletiva de sangue das veias adrenais para dosagens de aldosterona (22-25). Os aldosteronomas são tumores pequenos, em geral ao redor de 1 a $2 \mathrm{~cm}$, podendo ser menores e, mais raramente, maiores. A TC das adrenais é capaz de demonstrar a presença da maioria dos adenomas produtores de aldosteroma, exceto aqueles muito pequenos. Teoricamente, enquanto nos pacientes com APA nós podemos demonstrar um tumor unilateral, nos pacientes com HAI as glândulas aparecem com aspecto normal, discretamente hiperplasiadas ou com nódulos bilaterais.

Dos nossos 29 pacientes, 28 foram submetidos à TC ou RM e 2 ao mapeamento com iodo-colesterol, que é um método de imagem que não vamos comentar, porque não temos mais disponibilidade para rea- lizá-lo. Só devemos chamar a atenção de que este é um método de baixa sensibilidade, porém de maior especificidade. A maioria dos nossos pacientes tinha imagem positiva, principalmente na TC, que foi o principal método utilizado. A RM não parece ser superior à TC no diagnóstico radiológico dos tumores.

Quais são os problemas com o diagnóstico radiológico? Os tumores são pequenos e podem não ser detectados, além disso, pacientes com aldosteronoma podem ter outros nódulos adrenais que não participam da produção de aldosterona. Nesses casos, faz-se o diagnóstico equivocado de hiperplasia. Por outro lado, pacientes com HAI podem ter nódulos unilaterais na TC e, portanto, o diagnóstico de APA pode ser feito erroneamente. Finalmente, um incidentaloma adrenal não-funcionante pode ser detectado em um paciente portador de HAP causado por HAI, conduzindo o clínico ao diagnóstico equivocado de aldosteronoma (22-25). A nossa paciente é um exemplo de que nem sempre a identificação de um tumor adrenal pela TC confirma o diagnóstico de aldosteronoma, já que foi identificado um tumor adrenal à direita, que não era o responsável pela produção de aldosterona, como revelou o cateterismo e a evolução da paciente após a retirada desse tumor.

A maneira de solucionarmos essas armadilhas diagnósticas é com o cateterismo de veias adrenais. A informação fundamental fornecida por este exame é se a produção de aldosterona é unilateral ou bilateral. Atualmente, o cateterismo é considerado o padrãoouro no diagnóstico diferencial. Neste exame são colhidas, simultaneamente, amostras de sangue das veias adrenais direita e esquerda e da veia cava inferior (VCI) para determinações de aldosterona e cortisol. Consideramos os cateteres bem posicionados quando existe gradiente superior a 2 nos níveis de cortisol entre as adrenais e a periferia. Recomenda-se que se faça, durante o teste, a infusão de ACTH $(250 \mu \mathrm{g} \mathrm{em}$ $500 \mathrm{~mL}$ de soro fisiológico, na velocidade de 100 $\mathrm{ml} /$ hora) para minimizar eventuais alterações decorrentes da produção endógena de ACTH. Faz-se a determinação da aldosterona normalizada, que é o quociente aldosterona/cortisol, para corrigir os eventuais erros de diluição da amostra. O diagnóstico de APA é feito quando existe um gradiente maior ou igual a 4 entre as determinações da duas veias adrenais, sendo que no lado não-dominante a aldosterona é menor ou igual à da VCI, porque a produção desse lado deve estar bloqueada. No HAI, o gradiente entre adrenais é menor que 3 e a relação de aldosterona normalizada das veias adrenais com a VCI é maior ou igual a 1 . Um gradiente entre 3 e 4 é geralmente 
inconclusivo, mas pode ser conclusivo se a relação da aldosterona normalizada contra-lateral/VCI for inferior a 1 . Os pacientes com HPA têm comportamento semelhante ao APA no cateterismo.

Na nossa paciente, embora já tivéssemos estabelecido o diagnóstico de tumor adrenal à direita, houve certo questionamento se existia ou não aumento discreto da adrenal esquerda no exame de imagem. Em função desta dúvida, a paciente foi submetida ao cateterismo, que ocorreu sem nenhuma intercorrência e revelou produção unilateral de aldosterona à esquerda. Neste ponto da investigação, como o tumor à direita era óbvio e a lateralização no cateterismo também (embora do lado oposto), aventamos a possibilidade de ter ocorrido inversão acidental do material coletado. Como havia indicação da colecistectomia, decidimos pela exérese do tumor da adrenal direita no mesmo tempo cirúrgico. A retirada desse tumor não corrigiu, e nem mesmo melhorou, a hipertensão e a hipocalemia, o que confirmou que era um achado incidental, não envolvido na produção de aldosterona, e que o cateterismo localizou, com maior precisão, a produção de aldosterona. Na nossa experiência com os 29 pacientes portadores de aldosteronoma, a cirurgia corrigiu a hipocalemia em 100\% dos casos e hipertensão arterial em $48 \%$ deles, havendo necessidade de um número menor de medicações hipotensoras na maioria dos pacientes que não tiveram a normalização da pressão arterial no pós-operatório. Uma outra possibilidade é de que tivéssemos hiperplasia unilateral na adrenal esquerda e não aldosteronoma, e isso só poderia ser excluído ou confirmado após a retirada da adrenal esquerda, que não foi realizada. Como esta é uma eventualidade bem mais rara que o aldosteronoma, ficamos com a hipótese mais provável de um tumor adrenal esquerdo produtor de aldosterona. A possibilidade de hiperplasia bilateral é bastante remota, já que o diagnóstico histológico foi de adenoma na adrenal direita e, além disso, apesar de o diagnóstico diferencial histológico entre adenoma e hiperplasia nem sempre ser fácil, esperaríamos que uma adrenal, morfologicamente dominante, também apresentasse a mesma dominância funcional, o que não foi demonstrado pela sua retirada.

Essa paciente documentou a superioridade do cateterismo sobre os métodos de imagem na identificação do aldosteronoma. Atualmente, o cateterismo parece ser o padrão-ouro para o diagnóstico diferencial entre APA e HAI. Entretanto, devemos lembrar que, em cerca de $20 \%$ dos pacientes, ele não é bem sucedido por dificuldades técnicas, mesmo sendo realizado por profissionais experientes.
Em conclusão, uma vez feito o diagnóstico definitivo de HAP, deve-se proceder aos métodos radiológicos e, posteriormente, ao cateterismo toda vez que a radiologia for duvidosa ou não demonstrar tumor unilateral. Como foi sugerido por Espiner e col., mesmo na existência de tumor adrenal unilateral o cateterismo está indicado quando não existir queda na aldosterona concomitante com a queda do cortisol no teste postural de 4 horas (26). A queda do cortisol na postura sugere que a produção de aldosterona é dependente mais do ACTH do que do SRA e, portanto, compatível com um aldosteronoma. Esses autores, que realizam as dosagens de aldosterona e cortisol antes e após a adoção de postura ortostática, fazem as seguintes recomendações: 1) realizar a TC de adrenais: se existir um tumor nítido, maior que $1 \mathrm{~cm}$, unilateral, com a glândula contra-lateral normal, e a aldosterona diminuir no teste postural, o paciente não precisa ser submetido ao cateterismo; 2) se houver dúvida quanto à unilateralidade da lesão, ou seja, presença de tumor unilateral mas com a glândula contra-lateral aumentada, suspeita de doença bilateral, presença de tumor unilateral porém menor que $1 \mathrm{~cm}$ ou identificação de glândulas normais, deve-se realizar o cateterismo. Mesmo que haja tumor unilateral nítido, um teste postural com aumento ou manutenção da aldosterona deve indicar o cateterismo para diagnóstico definitivo. No cateterismo, se o gradiente entre as adrenais for maior ou igual a 4 com o gradiente entre o lado não-dominante e a VCI menor que 1 , faz-se o diagnóstico de produção unilateral de aldosterona, ou seja, aldosteronoma ou, mais raramente, HPA e recomenda-se o tratamento cirúrgico. Se o gradiente entre as adrenais for inferior a 3 e o gradiente entre o lado não-dominante e a VCI for maior ou igual a 1 , faz-se o diagnóstico de produção bilateral por provável HAI e recomenda-se o tratamento clínico. Um gradiente entre as adrenais entre 3 e 4 é inconclusivo; nesses casos, se o gradiente entre o lado não-dominante e VCI for menor que 1 , o diagnóstico mais provável é de APA e, se esse gradiente for maior que 1, o diagnóstico mais provável é de HAI.

A nossa paciente era portadora de tumor unilateral na TC sugerindo que ele fosse o aldosteronoma, embora o cateterismo não indicasse esse diagnóstico. Entretanto, a retirada cirúrgica desse tumor não corrigiu o HAP e permitiu a conclusão de que o cateterismo identificou o tumor com maior precisão do que a TC. Havia, portanto, um incidentaloma na adrenal direita e um aldosteronoma, não identificado radiologicamente, na adrenal esquerda. A paciente em discussão foi tratada clinicamente, com espironolactona e 
amlodipina, já que não quis ser submetida a outra cirurgia, e teve a hipocalemia e hipertensão corrigidas pelos uso dessas drogas.

Podemos concluir, pela apresentação desse caso clínico, que o cateterismo é o padrão-ouro no diagnóstico etiológico do HAP, como já foi sugerido por outros autores.

\section{DISCUSSĀO}

\section{Dr. Alexander Augusto de Lima Jorge}

Gostaria de saber se existe algum trabalho que tenha estudado a relação aldosterona/renina plasmática $(\mathrm{A} / \mathrm{R})$ e não aldosterona/atividade de renina plasmática (A/ARP).

\section{Dra. Debora Lucia Seguro Danilovic}

Existe. Não incluímos na apresentação para não nos estendermos demais. Um estudo demonstrou que a correlação entre as duas dosagens é bem semelhante $\mathrm{e}$ $\mathrm{o}$ valor da relação $\mathrm{A} / \mathrm{R}$ para o diagnóstico de HAP é de $5,4 \mathrm{ng} / \mathrm{dL} / \mathrm{ng} / \mathrm{L}$ (27). Nesse trabalho, os autores verificaram uma relação linear entre a atividade de renina plasmática e a renina. Da mesma forma, existe uma relação linear entre a A/ARP e a A/R. Talvez a utilização da dosagem de renina plasmática seja mais fácil porque diminui a interferência da qualidade da coleta do sangue, do período de incubação, e a influência da angiotensina plasmática.

\section{Dr. Jayme Goldman (Médico Assistente do Serviço de Endocrinologia e Metabologia do HCFMUSP)}

Depois dos trabalhos que demonstraram a ação extrarenal da aldosterona, você não considera que o tratamento clínico do HAP com drogas possa ser questionado?

\section{Dra. Debora Lucia Seguro Danilovic}

O tratamento com drogas, de modo geral, sim. Mas o tratamento específico, com espironolactona, que permite o bloqueio dos receptores mineralocorticóides renais e extra-renais, não. Já foi constatado que o uso deste medicamento em pacientes com insuficiência cardíaca diminui a morbi-mortalidade, provavelmente por diminuir a ação da aldosterona local, diminuindo fibrose, a expressão de PAI-l e o aumento de massa ventricular. O uso de outras drogas para o tratamento da HAS não deve ser eficaz na correção desses efeitos extra-adrenais da aldosterona. Portanto, acho que podemos concluir que a cirurgia é o melhor tratamento do APA e que, quando ela não é possível ou quan- do o paciente for portador de HAI, os antagonistas de receptores mineralocorticóides devem fazer parte, obrigatoriamente, do tratamento do HAP.

\section{Dr. Marcelo Cidade Batista (Médico Assis- tente do Serviço de Endocrinologia e Metabologia do HCFMUSP)}

Existe alguma padronização para a coleta de sangue para as determinações de aldosterona e atividade de renina plasmática?

\section{Dra. Debora Lucia Seguro Danilovic}

Não existe essa padronização. Muitos estudos nem citam como é feita essa coleta. Richard Gordon, autor que trabalha em centro de hipertensão na Austrália, sugere que a melhor maneira e hora de se fazer a coleta seria no período da manhã, com o paciente sentado há, pelo menos, 10 minutos após 2 horas de deambulação (28).

\section{Dra. Maria Adelaide Albergaria Pereira (Médica Assistente do Serviço de Endocrinologia e Metabologia do HCF- MUSP)}

É muito difícil a padronização da coleta, porque os vários laboratórios dão recomendações diferentes $\mathrm{e}$, como o paciente, em geral, está em investigação ambulatorial, o tempo que ele deambulou ou permaneceu sentado é muito difícil de ser controlado. Devemos ficar atentos tanto para a possibilidade da ocorrência de falsopositivos, que serão excluídos com os testes confirmatórios, mas também para a ocorrência de falso-negativos que podem ser provocados pelo uso da maioria dos agentes hipotensores. Como a Dra. Débora já disse, o estudo da relação A/ARP só serve para rastreamento e não serve para o diagnóstico. Pacientes com relação elevada podem não ter HAP, já que esta é uma fração cujo valor depende fundamentalmente do denominador, ou seja, da renina, que pode ser baixa em muitos pacientes com hipertensão essencial.

Quanto ao comentário do Dr. Jayme, chamamos a atenção que o tratamento clínico não pretende, somente, diminuir a PA, mas ele tem o objetivo de antagonizar, através do uso de inibidores dos receptores mineralocorticóides, os efeitos renais e extra-renais da aldosterona. $\mathrm{O}$ antagonismo das ações cardíacas da aldosterona deve ser fundamental na prevenção de doença cardíaca.

\section{Profa. Dra. Ana Claudia Latrônico Xavier (Médica Assistente do Serviço de Endocrinologia e Metabologia do HCF- MUSP)}


Eu tinha a informação de que uma relação A/ARP $>50$ poderia dispensar os testes confirmatórios para o diagnóstico de HAP. Também me parecia que, ao contrário do que você disse, o teste postural não tem papel importante no diagnóstico diferencial das várias causas de HAP.

\section{Dra. Debora Lucia Seguro Danilovic}

Como já dissemos, a relação A/ARP não tem especificidade para o diagnóstico, principalmente valores em torno de 50. Talvez números superiores a 200 possam ter maior especificidade, mas, de qualquer forma, é recomendável que todos os pacientes sejam submetidos aos testes confirmatórios após o rastreamento positivo. Devemos lembrar de não excluir o diagnóstico, definitivamente, em pacientes que fizeram o rastreamento na vigência do uso de drogas que elevam a renina e que, portanto, podem ter valor não-diagnóstico.

Quanto ao teste postural, ele de fato não é um método ideal para o diagnóstico diferencial. Os pacientes com aldosteronoma podem apresentar diminuição, aumento ou permanecer com os mesmos níveis de aldosterona durante a deambulação. O que os autores do trabalho que eu citei concluíram é que, quando existe diminuição da aldosterona com a postura, podemos concluir que o diagnóstico é de APA, já que a aldosterona segue o ritmo circadiano do ACTH. Se, nessas condições, a TC detectar o tumor, não é necessária a realização do cateterismo. Se ocorrer aumento ou manutenção da aldosterona com a mesma manobra, o diagnóstico diferencial não pode ser feito independentemente da existência ou não de tumor adrenal, e nesses casos é necessário que seja realizado o cateterismo adrenal.

\section{Prof. Dr. Geraldo Medeiros-Neto (Médico Assistente do Serviço de Endocrinologia e Metabologia do HCFMUSP)}

Você poderia dizer que as ações extra-renais da aldosterona são mais importantes do que aquelas sobre o epitélio do rim?

\section{Dra. Debora Lucia Seguro Danilovic}

Já foi verificado que os pacientes com hiperaldosteronismo têm alterações cardíacas, detectadas em exames de ecocardiograma e cintilografia miocárdica, mais importantes do que os pacientes com hipertensão essencial ou secundária a feocromocitoma ou síndrome de Cushing. Nós verificamos que o acometimento dos órgãos-alvo é muito importante no hiperaldosteronismo primário. Acho que podemos concluir que, provavelmente, as ações extra-renais da aldos- terona são tão importantes quanto as ações epiteliais renais.

\section{REFERÊNCIAS}

1. Kater CE. Rastreamento, comprovação e diferenciação laboratorial do hiperaldosteronismo primário. Arq Bras Endocrinol Metab 2002:46:106-15.

2. Ganguly A. Primary aldosteronism. N Engl J Med 1998; 339:1828-34.

3. Romagni P, Rossi P, Guerrini L, Quirini C, Santiemma V. Aldosterone induces contraction of the resistance arteries in man. Atherosclerosis 2003;166:345-9.

4. Dluhy RG, Williams GH. Aldosterone - villain or bystander? N Engl J Med 2004;351:8-10.

5. Pitt B, Zannad F, Remme Wj, Cody R, Castaigne A, Perez A, et al. The effects of spironolactone on morbidity and mortality in patients with severe heart failure: Randomized Aldactone Evaluation Study Investigators. N Engl J Med 1999; 341:709-17.

6. Rossi GP, Sacchetto A, Visentin PA, Graniero GR, Canali C, Pessina AC. Left ventricular mass and diastolic function in primary hiperaldosteronism and primary hypertension. Hypertension 1996;27:1039-45.

7. Rocha $R$, Rudolph $A E$, Frierdrich GE, Nachowiak DA, Kebec BK, Blomme EAG, et al. Aldosterone induces a vascular inflammatory phenotype in the rat heart. Am J Physiol 2002;283:1802-10.

8. Young MJ, Moussa L, Dilley R, Funder JW. Early inflammatory responses in experimental cardiac hypertrophy and fibrosis: effects of 11-hydroxysteroid dehydrogenase inactivation. Endocrinology 2003;144:1121-5.

9. Padfield PL. Prevalence and role of a raised aldosterone to renin ratio in the diagnosis of primary aldosteronism: a debate on the scientific logic of the use of the ratio in practice. Clin Endocrinol 2003;59:422-6.

10. Young Jr WF. Pheochromocytoma and primary aldosteronism: diagnostic approaches. Endocrinol Metab Clin North Am 1997:26:801-27.

11. Weinberger $\mathrm{MH}$, Fineberg NS. The diagnosis of primary aldosteronism and separation of the major subtypes. Arch Intern Med 1993;153:2125-9.

12. Montori VC, Schwartz GI, Chapman AB, Boerwinkle E, Turner ST. Validity of the aldosterone-renin ratio used to screen for primary aldosteronism. Mayo Clin Proc 2001;76:877-82.

13. Tanabe A, Naruse M, Takagi S, Tsuchiya K, Imaki T, Takano $\mathrm{K}$. Variability in the renin/aldosterone profile under random and standardized sampling conditions in primary aldosteronism. J Clin Endocrinol Metab 2003;88:2489-94.

14. Tiu SC, Choi Ch, Shek CC, Ng YW, Chan FKW, Ng CM, Kong APS. The use of aldosterone-renin ratio as a diagnostic test for primary hyperaldosteronism and its test characteristics under different conditions of blood sampling. J Clin Endocrinol Metab 2005;90:72-8.

15. Seifarth C, Trenkel S, Schobel H, Hahn EG, Hensen J. Influence of antihypertensive medication on aldosterone and renin concentration in the differential diagnosis of essential hypertension and primary aldosteronism. Clin Endocrinol 2002;57:457-65.

16. Mulatero $\mathrm{P}$, Rabbia F, Milan A, Paglieri C, Morello F, Chiandussi $L$, et al. Drug effects on aldosterone/plasma renin activity ratio in primary aldosteronsim. Hypertension 2002; 40:897-902.

17. Fardella CE, Mosso L, Gomez-Sanches CE, Cortés P, Soto J, Gómez L, et al. Primary hyperaldosteronism in essential hypertensives: prevalence, biochemical profile and molecular biology. J Clin Endocrinol Metab 2000;85:1863-7.

18. Mulatero P, Cella SM, Williams TA, Milan A, Mengozzi G, Chiandussi $L$, et al. Glucocorticoid remediable aldosteronism: low morbidity and mortality in a four-generation Italian pedigree. J Clin Endocrinol Metab 2002;87:3187-91.

19. Strauch B, Zelinka T, Hampf M, Bernhardt R, Widimsky Jr J. 
Prevalence of primary hyperaldosteronism in moderate to severe hypertension in the Central Europe region. J Hum Hypertens 2003; 17:349-52.

20. Young WF. Minireview: primary aldosteronism - changing concepts in diagnosis and treatment. Endocrinology 2003; $144: 2208-13$.

21. Mulatero P, Stowasser M, Loh KC, Fardella CE, Gordon RD, Mosso $L$, et al. Increase diagnosis of primary aldosteronism, including surgically correctable forms, in centers from five continents. J Clin Endocrinol Metab 2004;89:1045-50.

22. Stowasser M, Gordon RD. Prevalence and diagnostic workup of primary aldosteronism: new knowledge and new approaches. Nephrology 2001;6:119-26.

23. Doppman JL, Gill JR, Miller DI, Chang R, Gupta R, Friedman $\mathrm{TC}$, et al. Distinction between hyperaldosteronism due to bilateral hyperplasia and unilateral aldosteronoma: reliability of CT. Radiology 1992;184:677-82.

24. Magill SB, Raff H, Shaker JL, Brickner RC, Knechtges TE, Kehoe $\mathrm{ME}$, et al. Comparison of adrenal vein sampling and computed tomography in the differentiation of primary aldosteronism. J Clin Endocrinol Metab 2001;86:1066-71.

25. Rossi GP, Sacchetto A, Chiesura-Corona M, Toni R, Gallina M, Feltrin GP, et al. Identification of the etiology of primary aldosteronism with adrenal vein sampling in patients with equivocal computed tomography and magnetic resonance findings: Results in 104 Consecutive Cases. J Clin Endocrinol Metab 2001;86:1083-90.
26. Espiner EA, Ross DG, Yandle TG, Richards AM, Hunt PJ. Predicting surgically remedial primary aldosteronism: role of adrenal scanning, posture testing, and adrenal vein sampling. J Clin Endocrinol Metab 2003;88:3637-44.

27. Ferrari $P$, Shaw SG, Nicod J, Saner E, Nussberger J. Active renin versus plasma renin activity to define aldosterone-torenin ratio for primary aldosteonism. J Hypertens 2004;22:377-81.

28. Gordon RD. The challenge of more robust and reproducible methodology in screening for primary aldosteronism. J Hypertens 2004;22:251-5.

Endereço para correspondência:

Maria Adelaide Albergaria Pereira

Av. Dr. Enéas de Carvalho Aguiar 155, PAMB 8 andar, bloco 3 05403-000 São Paulo, SP

E-mail: adelaideap@uol.com.br 